\section{National Center for Sleep Disorders \\ Research}

Helga Peter

Marburg, Deutschland

\section{Definition}

Abteilung innerhalb des National Heart, Lung and Blood Institute (NHLBI) im Rahmen der National Institutes of Health (NIH) der USA.

\title{
Synonyme
}

NCSDR 